\title{
Estrategia para la optimización de un compuesto de caucho con aceite de soja utilizando diseños de experimentos
}

\author{
Strategy for the optimization of a rubber compound \\ with soybean oil using design of experiments
}

\author{
Pablo Ernesto Salvatori ${ }^{1,2}$, Gastón Rubén Sánchez ${ }^{2}$, \\ Aldo Gabriel Lombardi ${ }^{2}$, Esteban José Nicocia ${ }^{2}$, \\ Carlos Eugenio Boschetti ${ }^{1}$
}

\author{
${ }^{1}$ Area Tecnología Química, Instituto de Procesos Biotecnológicos y Químicos (IPROBYQ-CONICET) y Facultad de \\ Ciencias Bioquímicas y Farmacéuticas, Universidad Nacional de Rosario, Suipacha 531- 2000, Rosario, Santa Fé, Argen- \\ tina. \\ e-mail: psalvatori@outlook.com.ar; cboschet@fbioyf.unr.edu.ar \\ ${ }^{2}$ Desarrollo de Productos y Aplicaciones, Pampa Energía S.A, Av. Presidente Perón 1000 - 2202, Puerto General San \\ Martín, Santa Fe, Argentina. \\ e-mail: gaston.sanchez@pampaenergia.com; aldo.lombardi@pampaenergia.com; esteban.nicocia@pampaenergia.com
}

\section{RESUMEN}

Cuando se fabrica una pieza de goma, se parte de un "compuesto de caucho" que puede estar conformado por uno o más elastómeros y diferentes cargas. El desarrollo de una mezcla para elaborar un compuesto con un uso final específico es una tarea complicada en el ámbito de la tecnología del caucho. Por esto el desarrollo exitoso de un compuesto requiere de un trabajo sistemático siendo clave el uso de un diseño experimental estadístico (DoE).

Al implementar el DoE, deberán seleccionarse las variables para el sistema bajo investigación; las variables observadas o medidas (respuesta, propiedades del compuesto que desean controlarse) y las variables independientes que describen al sistema (los ingredientes y cantidades en la formulación del compuesto). El propósito del DoE es especificar el valor de las variables independientes, como también seleccionar la respuesta apropiada.

La estrategia DoE se ensayó anteriormente al utilizar dos grados de cauchos E-SBR (estireno-butadieno en emulsión), E-SBR 1712 y E-SBR 1721 y un caucho BR (polibutadieno) alto cis, con la finalidad de encontrar una mezcla de éstos que posea propiedades comparables a las de un neumático de alto rendimiento. En este trabajo se muestra el uso de la estrategia DoE para optimizar el agregado de un aceite extendedor al compuesto. Se utiliza una mezcla de tres tipos de aceites, dos aceites petroquímicos (Pq1 y Pq2) y un aceite vegetal de soja, buscando maximizar la cantidad de este último.

Las propiedades a ensayar se relacionan con características requeridas para la banda de rodamiento de un neumático: temperatura de transición vítrea $(\mathrm{Tg})$; relación entre módulo viscoso y módulo elástico (tan $@ 660^{\circ} \mathrm{C}$ ); Viscosidad Mooney y propiedades de tracción-elongación. Los valores obtenidos de cada propiedad se intentaron ajustar a modelos estadísticamente significativos, obteniéndose superficies de repuesta para cada una de ellas. Las superficies se combinan en la función deseabilidad, bajo ciertos criterios tanto para las propiedades buscadas, como también para la cantidad a usar de cada tipo de aceite en el compuesto óptimo deseable. Aplicando la función deseabilidad se logró predecir la composición óptima de aceites en el compuesto para alcanzar las propiedades deseadas, (3,17 pHR de aceite Pq1, 5,90 pHR de aceite Pq2 y 7,86 pHR de aceite de soja).

La validación satisfactoria de los modelos propuestos muestra la capacidad del DoE como estrategia útil para la optimización de propiedades en un compuesto de caucho de formulación compleja.

Palabras clave: Diseño de Experimentos, Materiales de caucho, Aceite vegetal.

\section{ABSTRACT}

When a rubber component is made, is necessary to start from a "rubber compound" which may be composed of one or more polymers and various additives or fillers. The development of an elastomer mixture to produce a compound with a specific end-use is complicated in the field of rubber technology. Therefore, the suc- 
cessful development of a compound requires a systematic work, being crucial to use a statistical design of experiments (DoE).

By implementing the DoE, the variables for the system under investigation should be selected; i.e., the observed or measured variables (responses, compound properties to be controlled) and the independent variables describing the system (the ingredients and amounts in the formulation of the compound). The purpose of the DoE is to specify the value of the independent variables, as well as to select the appropriate response.

The DoE strategy was already tested using two grades of E-SBR rubber (emulsion styrene-butadiene), E-SBR 1712, E-SBR 1721 and a BR rubber (polybutadiene) high-cis, in order to find a mixture of the three with properties comparable to those of a high performance tire. In this paper we will show the use of the DoE strategy working with the extender oil added to the compound. A mixture of three types of oils, two petrochemical oils ( $\mathrm{Pq} 1$ and $\mathrm{Pq} 2$ ) and soybean oil are used, seeking to maximize the amount of the latter.

The tested properties are those related to features required for the tread of a tire: the glass transition temperature $(\mathrm{Tg})$; the ratio between the viscous modulus and the elastic modulus $\left(\tan \delta @ 60^{\circ} \mathrm{C}\right)$; the Mooney Viscosity and the tensile-elongation properties. The values obtained for each measured property were fit to statistically significant models, yielding response surfaces for the properties. The surfaces are combined in the desirability function, where values are set for each property and under the condition that the amount of soybean oil is maximum in the mixture. Applying this desirability function, it was possible to predict the optimal composition of oils (3.17 pHq of oil Pq1, $5.90 \mathrm{pHR}$ of oil Pq2 and $7.86 \mathrm{pHR}$ of soybean oil) to be added to the compound to achieve the desired properties.

The successful experimental validation of the proposed models shows the ability of DoE as a useful strategy for the optimization of properties in a rubber compound with complex formulation.

Keywords: Design of Experiments, Rubber material, Vegetable oil.

\section{INTRODUCCIÓN}

La fabricación de un compuesto de goma es una tarea compleja en la industria cauchera, debido a la gran variedad de ingredientes que se utilizan, y por esto el desarrollo exitoso del material necesita de la implementación de un trabajo sistemático. El uso de un Diseño de Experimentos estadísticos (DoE) es clave para mejorar significativamente el proceso de desarrollo de un material, ya que puede usarse al DoE como estrategia para predecir y optimizar propiedades importantes para un determinado material de goma, obtenido a partir de la mezcla del elastómero con los distintos ingredientes en proporciones tabuladas dentro de una formulación. Utilizar un diseño estadístico en la industria del caucho, permitiría trabajar de una manera más ordenada y eficaz, dejando así de lado la metodología de prueba y error. Aplicando el DoE en mezclas complejas de caucho, se lograría un ahorro tanto en tiempo como en materiales y se conseguiría de manera más efectiva el material deseado [1-2].

El trabajo con la ayuda de un diseño experimental en el área de cauchos se ha mostrado en algunas publicaciones. Se puede mencionar, por ejemplo, su aplicación para evaluar y predecir las propiedades mecánicas finales de un caucho al variar las cargas y el sistema de vulcanización dentro de la formulación [1]; o con el objetivo de establecer una relación funcional entre la cantidad de negro de humo y aceite vegetal con diferentes propiedades del caucho, como también encontrar la cantidad óptima de aceite vegetal que funcione como acomplejante y permita la formación de enlaces químicos o físicos entre el negro de humo y el caucho [3]. También se ha utilizado el DoE para encontrar los parámetros adecuados en la polimerización de un BR alto cis. En este trabajo, se usaron las herramientas estadísticas para encontrar un eficiente sistema catalítico y las condiciones adecuadas de polimerización del butadieno [4].

En nuestro grupo hemos desarrollado anteriormente un trabajo donde se demostró la eficacia de la estrategia aquí propuesta, en la búsqueda de las mejores propiedades que se podían obtener con una mezcla ternaria de cauchos, dentro de una formulación de un compuesto típico para una banda de rodamiento de un neumático. En dicho caso se lograron optimizar y predecir propiedades finales específicas implementando el DoE junto con la función deseabilidad, la cual permite manipular múltiples respuestas [5]. La mezcla ternaria con que se trabajó contenía cauchos E-SBR 1712, E-SBR 1721 y BR alto cis; el uso del DoE permitió alcanzar valores deseables de propiedades importantes - $\tan \delta$ a $60{ }^{\circ} \mathrm{C}$ y Tg - que el caucho E-SBR 1712, principal componente de la banda de rodamiento de un neumático, por sí solo no posee.

Un tema que acapara atención en la industria neumatiquera desde hace un tiempo es el continuo desarrollo de productos que sigan manteniendo las altas prestaciones, o las mejoren, pero adaptándose a los requerimientos medioambientales que son cada vez más restrictivos. El reemplazo de diferentes componentes del neumático para cumplir con estos requerimientos ha dado lugar al desarrollo de una nueva generación de 
neumáticos, conocida como "Green Tyres". Los aceites extendedores, componentes fundamentales dentro de la formulación del neumático y que impactan considerablemente en las prestaciones finales, no escapan a estos desarrollos y regulaciones medioambientales. Como primer evento histórico en la regulación de los aceites extendedores, se encuentra la implementación de la Directiva Europea 2005/69/EC que prohíbe la utilización de aceites con alto contenido de compuestos policíclicos aromáticos (PCAs), que resultan cancerígenos, fijando límites en el orden de ppm para cada compuesto. Esto llevó a la industria a desarrollar nuevos aceites "no etiquetables", es decir que no poseen un rótulo de peligrosidad, y que son más amigables con el medio ambiente. Los aceites de "bajo PCAs" se pueden clasificar de acuerdo a su composición o también según el método de obtención. Estos aceites de bajo contenido de PCAs tienen una composición diferente a sus antecesores, lo cual afecta y además modifica las propiedades finales del material. La composición química de los aceites extendedores es muy importante a la hora de obtener una buena compatibilidad con el caucho [6].

Si bien a partir de las regulaciones mencionadas actualmente se utilizan sólo aceites con bajo contenido de PCAs, éstos son derivados del petróleo, un recurso no renovable. El poder reemplazar estos aceites petroquímicos, por aceites de origen vegetal ayudaría a disminuir la contaminación medioambiental y el posible problema de disponibilidad del petróleo. Esta iniciativa ya ha sido tomada en cuenta por diferentes grupos de investigación, que han evaluado el uso de un aceite derivado de alguna fuente renovable. Existen trabajos donde se utiliza aceite de soja en reemplazo del petroquímico, en los cuales se evalúa si el aceite vegetal es compatible con el caucho y cómo se ven afectadas las propiedades físicas de los compuestos obtenidos $[7,3]$.

En el presente trabajo se estudiará si se puede aplicar un diseño experimental que ayude a predecir y optimizar los valores de las propiedades más importantes para un neumático de alto rendimiento, en el cual se agregue como agente extendedor una mezcla de aceites que incorpore una cantidad máxima de aceite vegetal, teniendo en cuenta la tendencia a nivel mundial de poder reemplazar a los aceites petroquímicos por otros menos dañinos para el medio ambiente. La formulación de partida es la que se logró optimizar en un trabajo anterior [5], a la cual se le agrega en esta etapa una combinación de tres aceites: dos petroquímicos y uno vegetal. Así, se intentó evaluar cómo se ven afectadas las propiedades finales del compuesto con el aceite vegetal y la cantidad máxima de éste que se puede utilizar sin que se vean afectadas de manera negativa las propiedades del material.

\section{MATERIALES Y MÉTODOS}

\subsection{Formulación}

Se utilizó una formulación optimizada anteriormente, donde intervienen tres tipos de polímero. En la tabla 1 se muestran las características de los cauchos utilizados: dos cauchos estireno-butadieno, E-SBR 1712 y ESBR 1721 (Pampa Energía, Argentina) y un caucho de polibutadieno BR alto cis (Budene 1280, Goodyear, USA) [5].

Tabla 1: Características de los cauchos utilizados.

\begin{tabular}{l|l|l|l}
\hline TIPO DE CAUCHO & ESTIRENO (\%) & ACEITE (\%) & VISCOSIDAD MOONEY (MU) \\
\hline E-SBR 1712 & 23,5 & 27,5 & 53,7 \\
\hline E-SBR 1721 & 40,0 & 27,5 & 52,4 \\
\hline BR alto cis & 0 & 0 & 38,7 \\
\hline
\end{tabular}

MU: Unidades Mooney (arbitrarias)

La formulación mostrada en la tabla 2 contiene los cauchos mencionados, el sistema de acelerantes secundarios (óxido de zinc y acido esteárico), antioxidantes y antiozonantes (TMQ y DPPD) y el sistema vulcanizante (azufre, CBS y DPG); todos los componentes de la formulación fueron provistos por Pampa Energía (Argentina). Además, se agregan los tres aceites que forman parte del diseño, dos de origen petroquímicos: Aceite Pq1 (YPF, Argentina) y Aceite Pq2 (Nynas, Suecia) y uno de origen vegetal, de soja, (YPF). Las cantidades de cada uno se variarán en cada compuesto, de acuerdo a lo indicado por el diseño experimental, con el fin de encontrar la combinación óptima de los tres. En la tabla 3 se muestran las características de los aceites utilizados. 
Tabla 2: Formulación utilizada para todos los compuestos.

\begin{tabular}{l|l|l}
\hline COMPONENTE & PARTES (PHR) & MASA (G) \\
\hline E-SBR 1712 & 51 & 11,22 \\
\hline E-SBR 1721 & 66 & 14,52 \\
\hline BR alto cis & 45 & 9,90 \\
\hline OZn & 6,5 & 1,43 \\
\hline Ac. Esteárico & 2,7 & 0,59 \\
\hline TMQ & 2,7 & 0,59 \\
\hline DPPD & 2,7 & 0,59 \\
\hline Aceite del caucho E-SBR & 32 & 7,08 \\
\hline Aceite Pq1 & $*$ & $*$ \\
\hline Aceite Pq2 & $*$ & $*$ \\
\hline Aceite de Soja & $*$ & $*$ \\
\hline NH 330 & 91 & 20,02 \\
\hline CBS & 1,6 & 0,35 \\
\hline DPG & 0,4 & 0,09 \\
\hline Azufre & 2,4 & 0,53 \\
\hline Total & 292 & 64,24 \\
\hline & &
\end{tabular}

Tabla 3: Características de los aceites utilizados.

\begin{tabular}{l|l|l}
\hline TIPO DE ACEITE & CARACTERÍSTICAS & TG $\left({ }^{\circ} \mathbf{C}\right)$ \\
\hline Aceite Pq1 & Aromático con bajo contenido de PCA & $-56,24$ \\
\hline Aceite Pq2 & Nafténico con bajo contenido de PCA & $-44,38$ \\
\hline Aceite vegetal & Aceite de soja & $-44,50$ \\
\hline
\end{tabular}

\subsection{Diseño experimental}

El diseño experimental planteado en este trabajo pretende determinar la cantidad de cada uno de los aceites de la formulación, que proporcione propiedades optimizadas al compuesto de caucho. Se utilizó el programa Design Expert 7.0 (Stat-Ease Inc, USA), para desarrollar el diseño con el que se trabajó y poder encontrar la función deseabilidad adecuada.

Se utilizó un Diseño Central Compuesto (DCC) a tres factores, siendo éstos las cantidades (expresadas como pHR, "Parts per hundred rubber") de los diferentes aceites estudiados: pHR Pq1, pHR Pq2 y pHR soja. En total se prepararon 17 compuestos diferentes, cada uno con una combinación determinada de los tres aceites.

Los límites entre los cuales variar a cada factor se fijaron de acuerdo a las cantidades de aceite que se usan normalmente en el compuesto de un neumático. En una formulación de "Green Tyre" se agrega al compuesto hasta $45 \mathrm{pHR}$ de aceite [8]. Teniendo en cuenta que la formulación base (tabla 2) ya tiene $32 \mathrm{pHR}$ de aceite contenidos en los cauchos E-SBR, se decidió variar cada factor del diseño entre un mínimo de 0 pHR y un máximo de 10 pHR de cada aceite. Las cantidades resultantes se muestran en la tabla 4; en el diseño realizado puede observarse la cantidad total de aceite, suma de los tres agregados. Si además de este total se consideran las 32 pHR de aceite contenido en los cauchos E-SBR, resulta que el contenido total de aceite en todos los compuestos se encuentra entre 38 y 56 pHR, cubriendo así el rango que normalmente se usa en los neumáticos. 
Tabla 4: Combinaciones de los tres aceites.

\begin{tabular}{|c|c|c|c|c|}
\hline COMBINACIÓN & PQ1 (PHR) & PQ2 (PHR) & SOJA (PHR) & ACEITE TOTAL (PHR) \\
\hline 1 & 5 & 5 & 10 & 20 \\
\hline 2 & 10 & 5 & 5 & 20 \\
\hline 3 & 2,03 & 7,97 & 7,97 & 17,97 \\
\hline 4 & 5 & 10 & 5 & 20 \\
\hline 5 & 5 & 0 & 5 & 10 \\
\hline 6 & 2,03 & 2,03 & 7,97 & 12,03 \\
\hline 7 & 2,03 & 7,97 & 2,03 & 12,03 \\
\hline 8 & 5 & 5 & 0 & 10 \\
\hline 9 & 7,97 & 2,03 & 2,03 & 12,03 \\
\hline 10 & 5 & 5 & 5 & 15 \\
\hline 11 & 7,97 & 7,97 & 2,03 & 17,97 \\
\hline 12 & 7,97 & 7,97 & 7,97 & 23,91 \\
\hline 13 & 5 & 5 & 5 & 15 \\
\hline 14 & 7,97 & 2,03 & 7,97 & 17,97 \\
\hline 15 & 0 & 5 & 5 & 10 \\
\hline 16 & 2,03 & 2,03 & 2,03 & 6,09 \\
\hline 17 & 5 & 5 & 5 & 15 \\
\hline
\end{tabular}

\subsection{Preparación de los compuestos}

Los polímeros se mezclaron con las diferentes cargas en un molino Brabender Plasti-Corder a una temperatura de $60^{\circ} \mathrm{C}$ y velocidad del molino de $60 \mathrm{rpm}$. En una etapa previa se preparó, en un mezclador interno Banbury de $1250 \mathrm{~cm}^{3}$, una pre-mezcla consistente de los tres cauchos, más los acelerantes secundarios, la mitad del total de negro de humo a usar, los antioxidantes y antiozonantes, en cantidad suficiente para usar en todos los compuestos del diseño. Luego se utilizó la cantidad necesaria de esta pre-mezcla para cada carga en el molino Brabender. El ciclo de mezclado del Brabender se muestra en la tabla 5.

Tabla 5: Ciclo de mezclado en molino Brabender.

\begin{tabular}{l|l}
\hline TIEMPO & ACCIÓN \\
\hline Minuto 0 & Carga de la pre-mezcla, velocidad 30 rpm \\
\hline Minuto 1 & Agregado de la mezcla de aceites \\
\hline Minuto 2,5 & Carga del negro de humo restante, azufre, CBS y DPG \\
\hline Minuto 3 & Amasado a velocidad $60 \mathrm{rpm}$ \\
\hline Minuto 9 & Descarga del molino \\
\hline
\end{tabular}

Cada uno de los 17 compuestos con las cantidades detalladas en la tabla 2 se preparó por duplicado para obtener cantidad suficiente de material y poder así realizar todas las mediciones necesarias. Dichas mezclas duplicadas se juntaron y se homogenizaron en un molino abierto, manteniendo la temperatura de los rodillos en $45+/-5{ }^{\circ} \mathrm{C}$ durante toda la operación, de acuerdo a la norma ASTM D-3189.

\subsection{Medición de las propiedades}

Se cortaron porciones de cada compuesto obtenido para determinar la Viscosidad Mooney según ASTM D1646 con un instrumento Mooney MV 2000 (Alpha Technologies) y para medir las características de vulcanización según ASTM D-5289, se utilizó un Reómetro MDR2000 (Alpha Technologies) a $160{ }^{\circ} \mathrm{C}$ y con un arco de $0,5^{\circ}$.

Para obtener muestras de cauchos vulcanizados, cada compuesto se pasó por el molino una vez, obteniendo láminas de espesor 2,2 mm. (0,085"), las cuales se dejaron enfriar sobre una superficie plana metálica. Luego se cortó en planchas para vulcanizar y se marcó la dirección "del grano". Las planchas se vulcanizaron 
a $145^{\circ} \mathrm{C}\left(293^{\circ} \mathrm{F}\right)$ durante $22 \mathrm{~min}$, en una prensa a vapor, según ASTM D 3182. Terminada la vulcanización se acondicionaron las planchas vulcanizadas a temperatura de $23+/-3{ }^{\circ} \mathrm{C}$ durante 24 horas. Se cortaron probetas de las planchas vulcanizadas y se midieron las propiedades físicas: resistencia a la tracción, elongación y módulo 300\% (M300\%), según ASTM D-412, en un tensómetro T2000 (AlphaTechnologies). Para determinar la relación entre el módulo viscoso y elástico, tano, tanto del compuesto como del vulcanizado, se acondicionó el reómetro MDR 2000 [9]. Para la medición de propiedades térmicas, temperatura de transición vítrea $(\mathrm{Tg})$, se utilizó un calorímetro de barrido diferencial Jade DSC (Perkin Elmer).

\section{RESULTADOS}

En esta sección se enumeran los valores medidos de las diferentes propiedades y la función deseabilidad desarrollada para encontrar la composición óptima de aceites que permita obtener un compuesto con las propiedades deseables.

\subsection{Superficies de respuesta}

Los valores obtenidos para cada una de las propiedades medidas en cada uno de los 17 compuestos preparados se muestran en la tabla 6. Estos valores experimentales se pudieron ajustar a superficies de respuesta de cada propiedad, bajo modelos estadísticamente significativos. Estas superficies de respuesta, permiten evaluar cómo las propiedades se ven afectadas por cada uno de los factores en estudio, es decir, la cantidad de cada tipo de aceite.

Tabla 6: Valores de las propiedades en cada uno de los compuestos del diseño.

\begin{tabular}{|c|c|c|c|c|c|c|c|c|}
\hline \multirow[t]{2}{*}{ COMPUESTOS } & \multicolumn{8}{|c|}{ PROPIEDADES } \\
\hline & VM (MU) & TAN $\delta C$ & TAN $\delta \mathrm{V}$ & $\begin{array}{l}\text { TENSIL } \\
\text { (MPA) }\end{array}$ & $\begin{array}{l}\text { ELONGACIÓN } \\
\text { (\%) }\end{array}$ & $\begin{array}{l}\text { M300\% } \\
\text { (MPA) }\end{array}$ & $\begin{array}{l}\text { TG COMP } \\
\left({ }^{\circ} \mathrm{C}\right)\end{array}$ & $\begin{array}{l}\text { TG VULC } \\
\left({ }^{\circ} \mathrm{C}\right)\end{array}$ \\
\hline 1 & 42,6 & 0,456 & 0,211 & 19,67 & 682,87 & 6,42 & $-105,85$ & $-97,10$ \\
\hline 2 & 43,7 & 0,455 & 0,221 & 19,38 & 671,75 & 6,64 & $-105,84$ & $-97,13$ \\
\hline 3 & 43,5 & 0,454 & 0,212 & 18,80 & 626,38 & 7,03 & $-106,18$ & $-101,80$ \\
\hline 4 & 41,1 & 0,457 & 0,266 & 18,48 & 689,57 & 6,53 & $-105,19$ & $-98,15$ \\
\hline 5 & 52,2 & 0,441 & 0,223 & 19,40 & 586,32 & 8,38 & $-106,85$ & $-97,44$ \\
\hline 6 & 50,5 & 0,449 & 0,226 & 19,67 & 610,04 & 8,19 & $-105,53$ & $-98,81$ \\
\hline 7 & 50,5 & 0,445 & 0,213 & 18,80 & 686,45 & 8,50 & $-104,19$ & $-96,13$ \\
\hline 8 & 52,8 & 0,445 & 0,200 & 18,51 & 761,05 & 9,23 & $-106,18$ & $-97,82$ \\
\hline 9 & 48,0 & 0,449 & 0,208 & 18,81 & 687,61 & 8,47 & $-106,18$ & $-99,15$ \\
\hline 10 & 46,6 & 0,455 & 0,217 & 19,99 & 636,22 & 7,74 & $-106,18$ & $-98,47$ \\
\hline 11 & 45,3 & 0,453 & 0,213 & 19,91 & 610,10 & 7,48 & $-104,25$ & $-96,45$ \\
\hline 12 & 42,8 & 0,456 & 0,184 & 18,58 & 664,27 & 6,31 & $-104,15$ & $-95,12$ \\
\hline 13 & 46,6 & 0,450 & 0,220 & 19,29 & 625,49 & 7,62 & $-105,54$ & $-97,44$ \\
\hline 14 & 45,8 & 0,453 & 0,216 & 19,47 & 647,15 & 7,04 & $-105,27$ & $-94,80$ \\
\hline 15 & 51,1 & 0,453 & 0,207 & 19,69 & 611,61 & 8,75 & $-106,17$ & $-97,13$ \\
\hline 16 & 55,8 & 0,437 & 0,211 & 20,29 & 552,46 & 9,96 & $-106,55$ & $-98,80$ \\
\hline 17 & 49,3 & 0,456 & 0,211 & 19,08 & 624,11 & 7,53 & $-105,85$ & $-96,77$ \\
\hline
\end{tabular}

VM: Viscosidad Mooney. $\tan \delta \mathrm{C}$ : tangente delta del compuesto. tan $\delta$ V: tangente delta del vulcanizado. TG comp: Temperatura de transición vítrea del compuesto. TG vulc: Temperatura de transición vítrea del vulcanizado.

En el anexo A1, se muestran las superficies de respuesta de todas las propiedades evaluadas. Por cada propiedad medida se exponen dos figuras, mostrándose la variación en los valores de las propiedades en función de dos de los factores (pHR Pq1 y pHR de soja), manteniendo al factor pHR Pq2 en dos valores fijos: un valor bajo $(3,15 \mathrm{pHR})$ y otro alto $(7,57 \mathrm{pHR})$. 


\subsection{Función deseabilidad}

Teniendo la información de los valores de las ocho propiedades medidas en los compuestos, se pretende encontrar una combinación óptima de los factores (cantidad de cada aceite) que proporcione valores deseables para cada una de las ocho propiedades. Los valores deseables son los que debe presentar el compuesto de acuerdo a su uso final (neumático de alto rendimiento), discutidos en los siguientes párrafos. Se trata de un problema que presenta ocho respuestas, que varían al mismo tiempo cuando se modifican los factores; es por lo tanto un problema de optimización multi-respuesta. En términos matemáticos, la función deseabilidad logra una solución de compromiso entre los valores óptimos de cada una de las ocho propiedades. La superficie de cada una de las ocho propiedades se combina en la función deseabilidad, fijando un criterio para cada una de ellas, así como para la cantidad a usar de cada tipo de aceite en el compuesto óptimo deseable. A continuación, se detallan los criterios que se definieron en cada una de las ocho propiedades:

-La Viscosidad Mooney debe mantenerse dentro del rango en el cual variaron las muestras del diseño, es decir entre 41,1-55,8 MU.

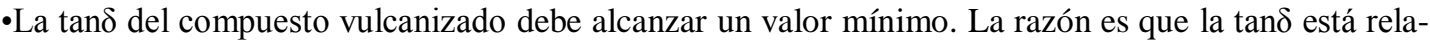
cionada con la resistencia al rodado (RR). Cuanto más bajo sea el valor de esta propiedad, menor la RR del neumático.

- La $\tan \delta$ del compuesto requiere un valor máximo. Esta propiedad se relaciona con la capacidad de moldeado del material por extrusión. Cuanto mayor sea este valor, más fácil será el extrudado.

-La resistencia a la tracción se pretende que llegue al máximo valor posible, ya que cuanto más alto, mayor la resistencia a la rotura del material final obtenido.

-Se priorizó un valor máximo del módulo 300\%, propiedad relacionada con la resistencia del material. Como la elongación es inversamente proporcional al módulo, se la fijó en un valor mínimo.

- La Tg, tanto del compuesto como del vulcanizado, deben tener valores bajos. Esta transición está relacionada con la resistencia a la rodadura, la tracción y el desgaste del neumático. Cuanto menor sea la $\mathrm{Tg}$, el material logrará un mayor rendimiento en condiciones de muy bajas temperaturas sin deteriorarse.

Fijados los criterios de optimización para las propiedades, se eligieron además los parámetros de la función deseabilidad de manera que la combinación de aceites óptima propuesta por el modelo se alcanzara con mínimos niveles de los dos aceites petroquímicos y se maximice el uso de aceite vegetal. Estas restricciones a las cantidades de los aceites posibilitan lograr el objetivo de reemplazar la mayor cantidad de aceite petroquímico por el vegetal. En la ecuación 1 se muestra la forma general de la función deseabilidad, donde $d_{i}$ representa cada respuesta transformada, que varía desde cero hasta uno; y D es la deseabilidad simultanea calculada como una media geométrica de todas las respuestas transformadas $d_{i}$. Las figuras 1a, 1b y 1c, muestran las superficies de respuesta de la función deseabilidad en función de dos de los factores, manteniendo fijo el tercer factor en su nivel óptimo. En la figura 1a se deja fijo el factor pHR soja, observándose valores altos de deseabilidad para cantidades menores de pHR Pq1 y altas de pHR Pq2. En la figura 1b el factor pHR Pq2 se mantiene fijo, lográndose valores máximos de deseabilidad con altas cantidades de pHR soja. Por último en la figura 1c, se deja fijo el factor pHR Pq1, mostrando que los valores más altos de deseabilidad se logran con altas cantidades de aceite de soja y $\mathrm{Pq} 2$.

$$
D=\left(d_{1} \times d_{2} \times \ldots \times d_{n}\right)^{\frac{1}{n}}=\left(\prod_{i=1}^{n} d_{i}\right)^{\frac{1}{n}}
$$




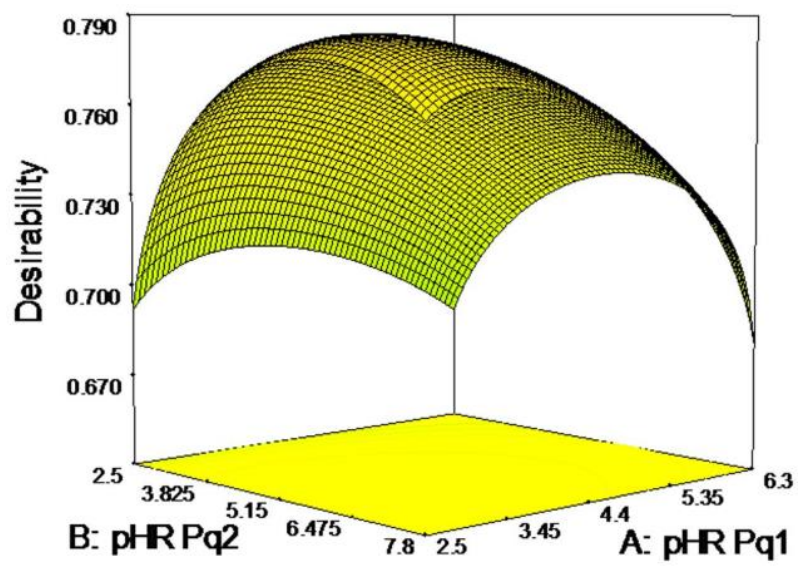

Figura 1a: Superficie para la función deseabilidad, manteniendo el factor pHR soja fijo en 7,86 pHR.

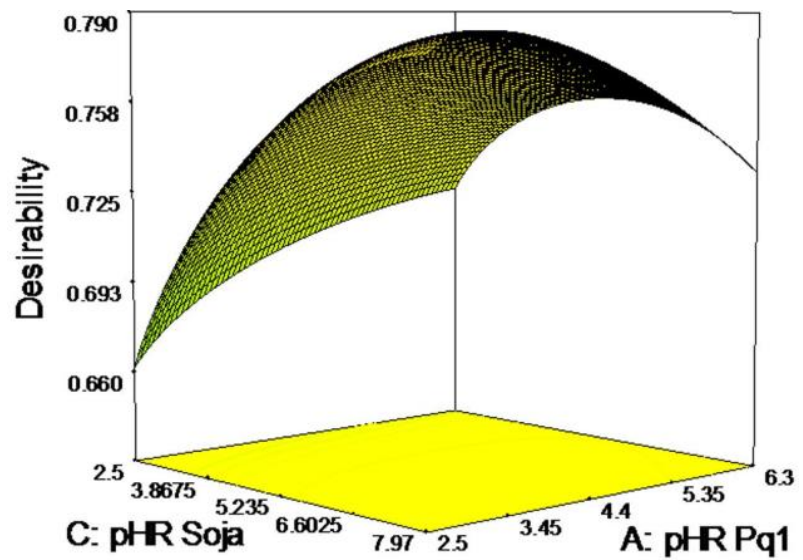

Figura 1b: Superficie para la función deseabilidad, manteniendo el factor pHR Pq2 fijo en 5,90 pHR.

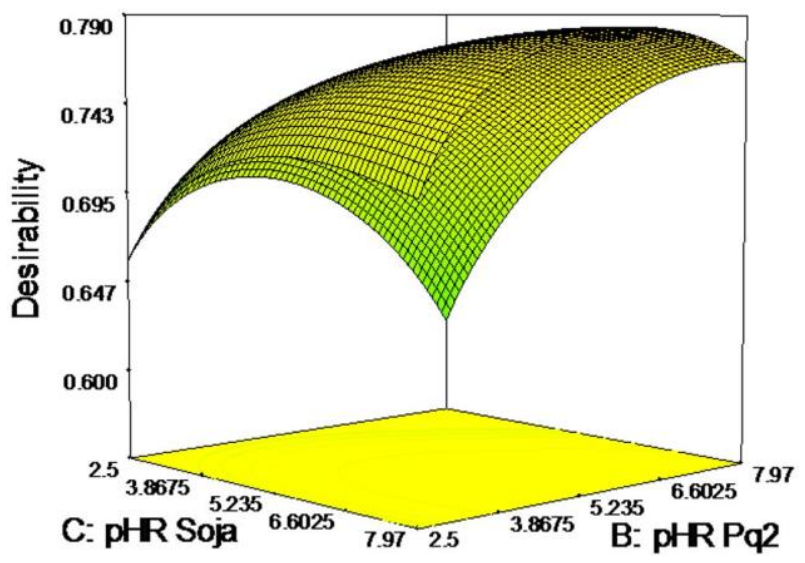

Figura 1c: Superficie para la función deseabilidad, manteniendo el factor pHR Pq1 fijo en 3,17 pHR.

A partir de la función deseabilidad, se obtuvieron los valores óptimos para cada uno de los tres factores: 3,17 pHR de aceite Pq1, 5,90 pHR de aceite Pq2 y 7,86 pHR de aceite de soja. Estas cantidades de cada tipo de aceite se introdujeron en la formulación de la tabla 2, para así preparar el compuesto con la mezcla de aceites óptima, aquella que permitirá obtener un material con los valores deseables de cada propiedad.

El total de aceite agregado a la mezcla resultó de 16,93 pHR, lo que sumado al ya contenido en los cauchos E-SBR (32 pHR), da un total de 48,93 pHR de aceite presente en la formulación del compuesto óptimo. Esta cantidad total de aceite resultante para el compuesto óptimo desarrollado es comparable a la que se suele usar en bandas de rodamiento para neumáticos [8]. 


\subsection{Mezcla optimizada.}

Para validar el modelo, se preparó por duplicado un compuesto con la mezcla óptima de los tres aceites, de acuerdo al resultado que arroja la función deseabilidad; luego sobre ambos compuestos se midieron cada una de las propiedades. En la tabla 7 se informan los resultados experimentales de las propiedades medidas, como promedio de los valores duplicados, junto con los valores predichos por el modelo construido.

Tabla 7: Comparación de valores predichos por el modelo y valores medidos de las diferentes propiedades.

\begin{tabular}{l|l|l}
\hline PROPIEDADES & PREDICHOS & MEDIDOS \\
\hline Viscosidad Mooney (MU) & 44,76 & $46 \pm 2$ \\
\hline $\tan \delta @ 60^{\circ} \mathrm{C}$ vulcanizado & 0,209 & $0,212 \pm 0,005$ \\
\hline $\tan \delta @ 60^{\circ} \mathrm{C}$ compuesto & 0,456 & $0,453 \pm 0,005$ \\
\hline Tensil (Mpa) & 19,2 & $19,6 \pm 0,8$ \\
\hline Elongación \% & 643 & $676 \pm 26$ \\
\hline $\mathrm{M} 300 \%(\mathrm{Mpa})$ & 7,2 & $6,7 \pm 0,3$ \\
\hline $\mathrm{Tg}\left({ }^{\circ} \mathrm{C}\right)$ compuesto & $-105,9$ & $-106,0 \pm 3,8$ \\
\hline $\mathrm{Tg}\left({ }^{\circ} \mathrm{C}\right)$ vulcanizado & $-99,2$ & $-100,0 \pm 3,8$ \\
\hline
\end{tabular}

\section{DISCUSIÓN}

Una etapa importante de la aplicación de DoE en una estrategia de optimización, es poder validar el modelo desarrollado. Esto se logra comparando los valores experimentales de las respuestas del problema optimizado, en este caso las ocho propiedades de los compuestos de cauchos, con los valores predichos por el modelo.

Observando la tabla 7, puede verse que la mayoría de los valores experimentales incluyen a los valores predichos por el modelo. Sin embargo, esto no se cumple en alguno de los casos. Por ejemplo, para las propiedades Elongación y Módulo al 300\%, los valores predichos están por fuera del rango del valor medido. En este caso, se sabe que ambas propiedades mantienen una relación inversa; esta correlación entre respuestas puede dificultar el proceso de optimización que requiere el ajuste de ambas respuestas en una única combinación de factores.

La estrategia de optimización presentada en este trabajo posibilitó determinar la cantidad máxima de aceite de soja que se puede usar en un compuesto de banda de rodamiento, sin que se vean afectados los valores esperados de cada propiedad del material. El uso de la mezcla de los tres aceites que cumple con todos los criterios fijados para cada propiedad, puede compararse con las propiedades obtenidas si se utiliza solo un tipo de aceite en el compuesto. Para esto se prepararon otros tres compuestos de acuerdo a la formulación de la tabla 2, agregando en cada caso un único aceite en una cantidad igual al total indicado por la combinación óptima (16,93 pHR). Sobre estos tres compuestos se midieron las ocho propiedades optimizadas. La tabla 8 muestra de manera gráfica cuáles son los casos en que se cumple el objetivo de mejorar la propiedad, comparando los compuestos con un aceite individual y el compuesto con la combinación óptima de aceites.

Tabla 8: Comparación entre la combinación óptima de los tres aceites y cada aceite de manera individual.

\begin{tabular}{|c|c|c|c|c|c|}
\hline \multirow[t]{2}{*}{ PROPIEDADES } & \multirow[t]{2}{*}{ OBJETIVOS } & \multicolumn{4}{|c|}{ COMPUESTOS } \\
\hline & & ACEITE PQ1 & ACEITE PQ2 & ACEITE SOJA & MEZCLA \\
\hline Viscosidad Mooney (MU) & En rango & $\checkmark$ & $\checkmark$ & $\checkmark$ & $\checkmark$ \\
\hline $\tan \delta @ 60^{\circ} \mathrm{C}$ vulcanizado & & $X$ & $\checkmark$ & $\mathrm{X}$ & $\checkmark$ \\
\hline $\tan \delta @ 60^{\circ} \mathrm{C}$ compuesto & & $X$ & $X$ & $\vee$ & $V$ \\
\hline Tensil(Mpa) & & $\checkmark$ & $\mathrm{X}$ & $X$ & $\checkmark$ \\
\hline Elongación \% & 1 & $\mathrm{X}$ & $\checkmark$ & $X$ & $V$ \\
\hline M300\% (Mpa) & & $\checkmark$ & $\mathrm{X}$ & $X$ & $\checkmark$ \\
\hline $\operatorname{Tg}\left({ }^{\circ} \mathrm{C}\right)$ compuesto & $\pi$ & $X$ & $\mathrm{X}$ & $\checkmark$ & $\checkmark$ \\
\hline $\operatorname{Tg}\left({ }^{\circ} \mathrm{C}\right)$ vulcanizado & 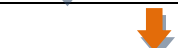 & $\mathrm{X}$ & $\mathrm{X}$ & $\vee$ & $\checkmark$ \\
\hline
\end{tabular}


A partir de la tabla 8 puede observarse que al trabajar con los aceites de manera individual no se logra un compuesto que alcance los objetivos deseados para el conjunto de todas las propiedades medidas. En esta comparación se verifica que los compuestos que poseen sólo un aceite de manera individual presentan valores aceptables para algunas propiedades, mientras que otras están fuera de los criterios fijados. Por el contrario, al trabajar con una mezcla óptima de los tres aceites, en una proporción que fue determinada con la ayuda del diseño de experimentos, se logra un compuesto que cumple con el objetivo buscado en cada una de las propiedades medidas. Si bien el compuesto con la mezcla de aceites optimizada puede no alcanzar mínimos o máximos absolutos en alguna propiedad, dicha mezcla posibilita reunir todas las características positivas de cada aceite, compensando las negativas.

\section{CONCLUSIONES}

En este trabajo se pudo mostrar que el uso del diseño de experimentos es una herramienta eficaz para la elaboración de un nuevo material con propiedades específicas, lo que permite un trabajo más ordenado y eficiente, con ahorro tanto en tiempo como en materiales.

En el caso de los materiales de caucho aquí presentados, se llevó adelante un diseño en el que se estudió una mezcla de tres tipos diferentes de aceites, siendo uno de ellos de origen vegetal. Se lograron optimizar ocho propiedades del material de caucho, importantes para su aplicación en la banda de rodamiento de un neumático. El diseño empleado permitió definir una combinación óptima de los aceites, en la cual se priorizó el uso del aceite de soja por sobre los aceites de origen petroquímico. La combinación de aceites definida por el diseño se pudo validar preparado el compuesto optimizado y corroborando que los valores experimentales fueron comparables a los predichos, siendo compatibles a los que se esperan en un neumático de alta performance.

\section{AGRADECIMIENTOS}

Los autores agradecen a CONICET (PIP No 179CO) y a Pampa Energía S.A, por el financiamiento parcial de este trabajo. P.E.S. agradece a CONICET y a Pampa Energía S.A por una beca cofinanciada para realizar su tesis doctoral. Se agradece a Pampa Energía S.A por permitir publicar resultados obtenidos parcialmente en su Planta de Puerto General San Martín (Argentina).

\section{BIBLIOGRAFÍA}

[1] BALACHANDRAN, M., BHAGAWAN, S.S, MURALEEKRISHNAN, R., "Design of experiments for optimizing NBR nanocomposite formulations", In: International Conference on Advance in Polymer Technology, p. 82, Cochin, India, Sep. 2008.

[2] SOMMERS, J.G., "Elastomers and Compounds", In: Engineered Rubbers Products. Introduction to Design, Manufacture and Testing, 1 ed., capitulo 2, Munich, Hanser Publishers, 2009.

[3] KUKREJA, T.R., KUMAR, D., PRASAD, K., et al., "Optimisation of physical and mechanical properties of rubber compounds by response surface methodology-Two component modeling using vegetable oil and carbon black", European Polymer Journal, v. 38, n. 7, pp. 1417-1422, Jul. 2002.

[4] MADHUCHHANDA, M., SRIVASTAVA, V. K., SHEWALE, S., et al., "Process parameter optimization through Design of Experiments in synthesis of high cis-polybutadiene rubber", Chemical Engineering Science, v. 107, pp. 256-265, Abr. 2014.

[5] SALVATORI, P.E, SÁNCHEZ, G., LOMBARDI, A., et al., "Desarrollo de superficies de respuesta para la optimización de propiedades en una mezcla ternaria de caucho", Simposio Argentino de Polímeros, Santa Fe, 20-23 Oct. 2015.

[6] MONTEALEGRE GARCÍA, G., "Extensores (plastificantes) aromáticos no etiquetables: altas prestaciones para la industria del neumático", Revista SLTCAUCHO Industria y Tecnología en América Latina, n. 12, pp. 24-31, Abr. 2016.

[7] PETROVÍC ZORAN, S., IONESCU, M., MILÍC, J., et al., "Soybean Oil Plasticizers as Replacement of Petroleum Oil in Rubber”, Rubber Chemistry and Technology, v. 86, n. 2, pp. 233-249, Jun. 2013.

[8] FERNÁNDEZ, M., "Mejores propiedades con el uso de BUNA CB en calzados, bandas de rodamiento y cintas transportadoras”, Lanxess Tech Series, Bogotá, 29 Ago. 2012.

[9] RAMPERTI, A., "Control Reológico: Visión integral ¿Y si no poseo un RPA?”, Revista SLTCAUCHO Industria y Tecnología en América Latina, n. 13, pp. 8-16, Jun. 2016. 